\title{
LISTA DE PARECERISTAS AD HOC DE 2018
}

o final de mais um ano, os Editores Associados da revista Educação \& Sociedade e a Diretoria do Centro de Estudos Educação e Sociedade

(Cedes) agradecem a colaboração de todos os membros de seus conselhos editoriais e colaboradores permanentes e, particularmente, aos pareceristas ad hoc elencados a seguir, que emitiram pareceres e promoveram, com sua experiência e profissionalismo, a arbitragem dos textos submetidos para avaliação neste periódico, durante o ano de 2018.

Adilson Dalben

Sesi-SP de Educação, Brasil

Adolfo Ignacio Calderón

Pontifícia Universidade Católica de

Campinas, Brasil

\section{Adriana Bauer}

Universidade de São Paulo, Brasil

\section{Alberto Moreno-Dońa}

Escuela de Educación Parvularia, Universidad de Valparaíso, Chile

\section{Alda Junqueira Marin}

Pontifícia Universidade Católica de

São Paulo, Brasil

\section{Alexandro HenriquePaixáo}

Universidade Estadual de Campinas, Brasil

\section{Almerico Lima}

Universidade Federal da Bahia, Brasil

Alvanize Valente Fernandes Ferenc

Universidade Federal de Viçosa, Brasil

Amurabi Oliveira

Universidade Federal de Santa

Catarina, Brasil
Ana Luiza Bustamante Smolka

Universidade Estadual de Campinas, Brasil

\section{Ana Maria Saul}

Pontifícia Universidade Católica de

São Paulo, Brasil

\section{Anderson Ricardo Trevisan}

Universidade Estadual de Campinas, Brasil

\section{Ângela Maria Martins}

Fundação Carlos Chagas, Brasil

\section{Angela Scalabrin Coutinho}

Universidade Federal do Paraná, Brasil

\section{Angelica Cosenza}

Universidade Federal de Juiz de Fora, Brasil

\section{Antonio Carlos Rodrigues Amorim} Universidade Estadual de Campinas, Brasil
Antonio Henrique Pinto
Instituto Federal de Educação
Tecnológica do Espírito Santo, Brasil 
Antonio Medina Rivilla

Universidad Nacional de Educación a

Distancia, Espanha

Antonio Soares

Instituto Federal do Rio de Janeiro,

Brasil

Aparecida da Silva Xavier Barros

Instituto Federal da Paraíba, Brasil

Benedita Portugal e Mello

Instituto de Educação, Universidade

de Lisboa, Portugal

Bernardo Mançano Fernandes

Faculdade de Ciências e Tecnologia,

Universidade Estadual Paulista "Júlio

de Mesquita Filho", Brasil

Camila Ferreira da Silva

Universidade Nova de Lisboa, Portugal

Carla Carrochano

Universidade Federal de São Carlos, Brasil

Carmen Elvira F. Mendoza Prado

Universidade Federal de Minas Gerais, Brasil

\section{Cassia Domiciano}

Centro Universitário São Camilo, Brasil

Clarice Seixas Duarte

Universidad Politécnica de Madrid,

Espanha

\section{Clarissa Eckert Baeta Neves}

Universidade Federal do Rio Grande

do Sul, Brasil

Cláudio Marques Martins Nogueira

Universidade Federal de Minas Gerais,

Brasil

Cristiane Machado

Universidade Estadual de Campinas, Brasil
Cristina Sendra Mocholí

Universitat de València, Espanha

Daniel Ferraz Chiozzini

Pontifícia Universidade Católica de

São Paulo, Brasil

\section{Debora Goulart}

Universidade Federal de São Paulo, Brasil

\section{Deise Mancebo}

Universidade do Estado do Rio de Janeiro, Brasil

\section{Dilmeire Sant'Anna}

Ramos Vosgerau

Pontifícia Universidade Católica do

Paraná, Brasil

\section{Dirce Pacheco Zan}

Universidade Estadual de Campinas, Brasil

\section{Divino José da Silva}

Universidade Estadual Paulista "Júlio de Mesquita Filho", Brasil

\section{Eldon Henrique Mühl}

Universidade de Passo Fundo, Brasil

Elenice Maria Cammarosano Onofre

Universidade Federal de São Carlos, Brasil

\section{Eliana Lucia Madureira Yunes}

Pontifícia Universidade Católica do

Rio de Janeiro, Brasil

\section{Elie George Guimarães}

Ghanem Júnior

Universidade de São Paulo, Brasil

Elizabeth Macedo

Universidade do Estado do Rio de Janeiro, Brasil

\section{Elmir de Almeida}

Universidade de São Paulo, Brasil 


\section{Estela Costa}

Instituto de Educação, Universidade de Lisboa, Portugal

\section{Ester Rizzi}

Universidade de São Paulo, Brasil

\section{Fabiana de Cássia Rodrigues}

Universidade Estadual de Campinas, Brasil

\section{Fátima Antunes}

Universidade do Minho, Portugal

\section{Felicitas Acosta}

Universidad Nacional de General

Sarmiento, Argentina

\section{Felipe Quintão Almeida}

Universidade Federal do Espírito

Santo, Brasil

\section{Fernando Luiz Cássio}

Fundação Universidade Federal do ABC, Brasil

\section{Fernando Perlatto}

Universidade Federal de Juiz de Fora, Brasil

\section{Flaviany Ribeiro Silva}

Universidade do Estado do Rio de Janeiro, Brasil

\section{Francisco Heitor De Magalhães}

Souza

Universidade Estadual de Goiás, Brasil

\section{Gabriela Lotta}

Escola de Administração de Empresas, Fundação Getulio Vargas, Brasil

\section{Gisele Masson}

Universidade Estadual de Ponta

Grossa, Brasil

\section{Gladys Beatriz Barreyro}

Universidade de São Paulo, Brasil

\section{Gustavo Melo-Silva}

Universidade Federal de São João

Del-Rei, Brasil

\section{Helena Reis Cabeleira}

Universidade de Lisboa, Portugal

\section{Helena Troiano}

Universidade Aberta do Brasil, Brasil

\section{Jaime Giolo}

Universidade de Passo Fundo, Brasil

\section{Jane Bittencourt}

Universidade Federal de Santa

Catarina, Brasil

\section{Janete Maria Lins de Azevedo}

Universidade Federal de Pernambuco, Brasil

\section{Jefferson Mainardes}

Universidade Estadual de Ponta

Grossa, Brasil

\section{Jessica Garabal-Barbeira}

Universidade da Coruña, Espanha

José Carlos Rothen

Universidade Federal de São Carlos, Brasil

\section{Jose Francisco Soares}

Universidade Federal de Minas Gerais, Brasil

\section{José Ignacio Rivas Flores}

Universidad de Málaga, Espanha

José Leon Crochik

Universidade de São Paulo, Brasil

José Ossian Gadelha Lima

Faculdade de Educação de Crateús,

Universidade Estadual do Ceará, Brasil

\section{José Pedro Boufleuer}

Universidade Regional do Noroeste do

Estado do Rio Grande do Sul, Brasil 
José Sánchez Santamaría

University of Castilla-La-Mancha, Espanha

Juan Antonio Morales Lozano

Universidad de Sevilla, Espanha

Juan Pablo Valenzuela

Universidad de Chile, Chile

Judith Jacovkis Halperin

Universidad Autónoma de Barcelona, Espanha

Juliana Campregher Pasqualini

Universidade Estadual Paulista "Júlio de Mesquita Filho", Brasil

Kimi Tomizaki

Universidade de São Paulo, Brasil

Lais Silveira Fraga

Universidade Estadual de Campinas, Brasil

\section{Leandro Lajonquière}

Universidade de São Paulo, Brasil

\section{Leda Maria Caira Gitahy}

Universidade Estadual de Campinas, Brasil

Leda Scheibe

Universidade Federal de Santa

Catarina, Brasil

\section{Leonor L. Torres}

Universidade de Minho, Portugal

\section{Lourdes de Fátima B. Carril}

Universidade Federal de São Carlos, Brasil

\section{Lucia Leite}

Universidade Estadual Paulista "Júlio de Mesquita Filho", Brasil

\section{Lúcia Mercês de Avelar}

Universidade Estadual de Campinas, Brasil

\section{Lucia Pintor Santiso Villas Bôas}

Fundação Carlos Chagas, Brasil

\section{Luciana Azevedo Rodrigues}

Universidade Federal de Lavras, Brasil

\section{Luciana Rosa Marques}

Universidade Federal de Pernambuco, Brasil

\section{Luciane Muniz Ribeiro Barbosa}

Universidade Estadual de Campinas,

Brasil

\section{Lucilia Augusta Lino}

Universidade Estadual do Rio de Janeiro, Brasil

\section{Luis Armando Gandin}

Universidade Federal do Rio Grande do Sul, Brasil

\section{Luis Enrique Aguilar}

Universidade Estadual de Campinas, Brasil

\section{Luis Marcelo Carvalho}

Universidade Estadual Paulista "Júlio de Mesquita Filho", Brasil

\section{Luiz Antônio Calmon Nabuco}

\section{Lastória}

Universidade Estadual Paulista "Júlio de Mesquita Filho", Brasil

\section{Luzia Batista de Oliveira Silva}

Universidade São Francisco, Brasil

Manuel Jacinto Sarmento

Universidade do Minho, Portugal

Márcia Aparecida Jacomini

Faculdade Anhanguera, Brasil

Marcia de Paula Leite

Universidade Estadual de Campinas, Brasil 
Marcia R. Erica Castilho Rodrigues

Universidade Federal de Ouro Preto,

Brasil

\section{Maria Amália Almeida Cunha}

Universidade Federal de Minas Gerais, Brasil

\section{Maria Antônia de Souza}

Universidade Estadual de Ponta

Grossa, Brasil

\section{Maria Cecília Rafael Góes}

Universidade Metodista de Piracicaba, Brasil

Maria Cristina Fernandes

Universidade Federal de São Carlos, Brasil

\section{Maria da Graça Bolman \\ Universidade do Sul de Santa \\ Catarina, Brasil}

\section{Maria Eugénia Ferráo}

Universidade da Beira Interior, Portugal

Maria Giudicissi Rehder Rehder

Universidade de São Paulo, Brasil

María Elena Mellado-Hernández

Universidad Católica de Temuco, Chile

Maria Helena Oliveira Gonçalves

\section{Augusto}

Universidade Federal de Minas Gerais, Brasil

\section{Maria Isabel da Cunha}

Universidade Federal de Pelotas

Maria José Casa Nova

Universidade do Minho, Portugal

Maria Lúcia Büher Machado

Instituto Federal de Educação, Ciência

e Tecnologia do Paraná, Brasil
Maria Luiza Rodrigues Flores

Universidade Federal do Rio Grande do Sul, Brasil

\section{Maria-Beatriz Moreira Luce}

Universidade Federal do Rio Grande do Sul, Brasil

\section{Mariana Moreno Castilho}

Universidade de São Paulo, Brasil

\section{Mariângela Graciano}

Universidade Federal de São Paulo, Brasil

Marilda da Silva

Universidade Estadual Paulista "Júlio de Mesquita Filho", Brasil

\section{Mario Lopes Amorim}

Universidade Tecnológica Federal do

Paraná, Brasil

\section{Mauro Carlos Moschetti}

Universitat Autònoma de Barcelona, Espanha

\section{Mauro Mediavilla}

Universitat de València, Espanha

\section{Miguel Russo}

Universidade Cidade de São Paulo, Brasil

\section{Nadja Hermann}

Conselho Nacional de

Desenvolvimento Científico e

Tecnológico, Brasil

\section{Naira Lisboa Franzoi}

Universidade Federal do Rio Grande do Sul, Brasil

\section{Nalu Farenzena}

Universidade Federal do Rio Grande do Sul, Brasil

\section{Nelson Cardoso do Amaral}

Universidade Federal de Goiás, Brasil 
Nilo Agostini

Universidade São Francisco, Brasil

Nilson Marcos Dias Garcia

Universidade Tecnológica Federal do

Paraná, Brasil

Ofelia Roldán Vargas

Fundación Centro Internacional de

Educación y Desarrollo Humano,

Colômbia

Olinda Evangelista

Universidade Federal de São Carlos,

Brasil

\section{Patrícia Vieira Trópia}

Universidade Federal de Uberlândia, Brasil

\section{Pedro Caetano}

Universidade Nova Lisboa, Portugal

Philippe Pomier Layrargues

Universidade de Brasília de Planaltina, Brasil

\section{Raquel Fontes Borghi}

Universidade Estadual Paulista "Júlio de Mesquita Filho", Brasil

\section{Regina Tereza Cestari de Oliveira}

Universidade Católica Dom Bosco, Brasil

\section{Robert Verhine}

Universidade Federal da Bahia, Brasil

\section{Roberto Gomes}

Universidade Federal de São Carlos, Brasil

\section{Robson Loureiro}

Universidade Federal do Espírito Santo, Brasil

\section{Rodrigo Rosistolato}

Universidade Federal do Rio de Janeiro, Brasil
Ronaldo Marcos de Lima Araujo

Universidade Federal do Pará, Brasil

Rosa Maria da Exaltaçáo Coutrim

Universidade Federal de Ouro Preto, Brasil

\section{Rosana Evangelista da Cruz}

Universidade Federal do Piauí, Brasil

Roseli Esquerdo Lopes

Universidade Federal de São Carlos, Brasil

Rubens Barbosa de Camargo

Universidade de Sáo Paulo, Brasil

\section{Ruth Pinedo}

Universidad de Valladolid, Espanha

\section{Sabrina Moehlecke}

Universidade Federal do Rio de Janeiro, Brasil

\section{Sebastian Donoso}

Universidad de Talca, Chile

Sérgio Antonio da Silva Leite

Universidade Estadual de Campinas, Brasil

\section{Shirley Silva}

Universidade de São Paulo, Brasil

\section{Sinésio Ferraz Bueno}

Universidade Estadual Paulista "Júlio de Mesquita Filho", Brasil

\section{Sofia Viseu}

Universidade de Lisboa, Portugal

Sônia Maria da Silva Araújo

Universidade Federal do Pará, Brasil

\section{Tatiane Cosentino Rodrigues}

Universidade Federal de São Carlos, Brasil

Teise Oliveira Guaranha Garcia

Universidade de São Paulo, Brasil 


\section{Thiago Duarte Pimentel}

Universidade Federal de Juiz de Fora, Brasil

\section{Toni Reis}

Universidade do Vale do Rio dos Sinos, Brasil

\section{Valéria Virginia Lopes}

Consultora educacional

\section{Vanda Mendes Ribeiro}

Universidade de São Paulo, Brasil

\section{Vanessa Elias de Oliveira}

Fundação Universidade Federal do ABC, Brasil

\section{Vanessa Lima Caldeira Franceschini}

Universidade Federal de Minas Gerais, Brasil

\section{Wilson Mesquita de Almeida}

Fundação Universidade Federal do ABC, Brasil 